\title{
Two Cases of an Epidermoid Cyst Developing in an Intrapancreatic Accessory Spleen Identified during Laparoscopic Distal Pancreatectomy
}

\author{
Masakuni Fujii, Masao Yoshioka and Junji Shiode
}

\begin{abstract}
Epidermoid cysts presenting within an intrapancreatic accessory spleen are rare non-neoplastic cysts typically occurring in the pancreatic tail. This entity is difficult to diagnose given there are many types of pancreatic neoplastic cysts. We herein describe two cases of an epidermoid cyst within an intrapancreatic accessory spleen for which we performed a resection by laparoscopic distal pancreatectomy. Epidermoid cysts in an intrapancreatic accessory spleen should therefore be considered in the differential diagnosis of pancreatic tail cystic lesions. Laparoscopic distal pancreatectomy can be a useful, minimally invasive surgical approach for treating these cysts as well as for the treatment of benign or low-grade malignant tumors located in the pancreatic body or tail.
\end{abstract}

Key words: epidermoid cyst, intrapancreatic accessory spleen, laparoscopic distal pancreatectomy

(Intern Med 55: 3137-3141, 2016)

(DOI: 10.2169/internalmedicine.55.7141)

\section{Introduction}

The occurrence of an accessory spleen is not rare; it affects approximately $10 \%$ of the general population, and from $16-20 \%$ of all cases are intrapancreatic $(1,2)$. However, an epidermoid cyst in an accessory spleen within the pancreas is rare. Differentiating an epidermoid cyst from other cystic neoplasms of the pancreas is difficult but important for selecting the appropriate management. Contrast enhanced computed tomography (CT) and magnetic resonance image (MRI) can aid in preoperatively identifying these cysts based on the characteristic imaging features. We experienced two cases of an epidermoid cyst occurring within an intrapancreatic accessory spleen. Given this rare presentation of epidermoid cysts, we initially favored a diagnosis of intraductal papillary mucinous neoplasms (IPMN) or mucinous cystic neoplasms (MCN). Therefore we opted for a conservative approach by resecting the lesions in both patients through a laparoscopic distal pancreatectomy. Retrospectively, we found few reports of this type of cyst, with little in terms of characteristic imaging findings to help guide management. Laparoscopic distal pancreatectomy can be a useful procedure, with the advantages of low invasiveness, for the treatment of benign or low-grade malignant tumors located in the pancreatic body or tail.

\section{Case Reports}

\section{Case 1}

An asymptomatic woman in her 50s had a cystic lesion in the tail of the pancreas identified by abdominal ultrasonography (US) during a medical examination. She was hospitalized at our institution for further workup of this cystic lesion in the pancreas. Her medical history included an appendectomy in her 20s. Physical examination was essentially unremarkable. Laboratory data showed a slightly elevated carbohydrate antigen $19-9$ of $43.1 \mathrm{U} / \mathrm{mL}$ (normally $\leq 37 \mathrm{U} / \mathrm{mL}$ ). Abdominal US showed a $40 \mathrm{~mm}$ unilocular cystic lesion of the pancreatic tail. The borders were clear with heterogenous echogenicity. There were no mural nodules in the cystic lesion. A contrast enhanced abdominal CT scan confirmed the presence of a $40 \mathrm{~mm}$ unilocular cystic lesion 

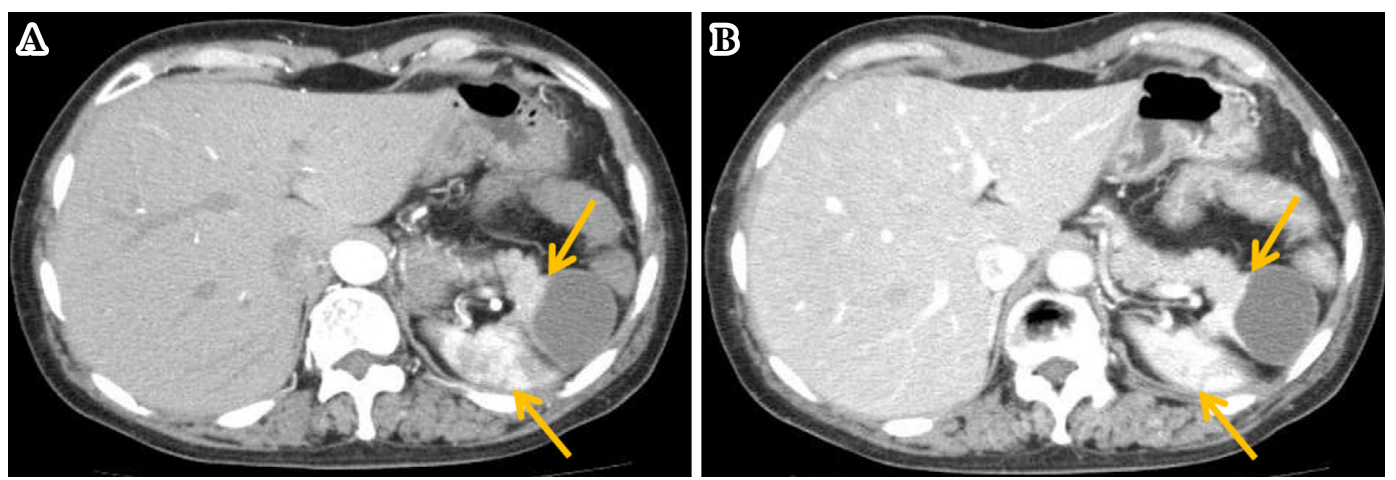

Figure 1. The contrast enhanced abdominal computed tomography (CT) scan confirmed a $40 \mathrm{~mm}$ unilocular cystic lesion of the pancreatic tail, which showed enhancement similar to the adjacent spleen (arrows) (artery phase) (A), (portal phase) (B).

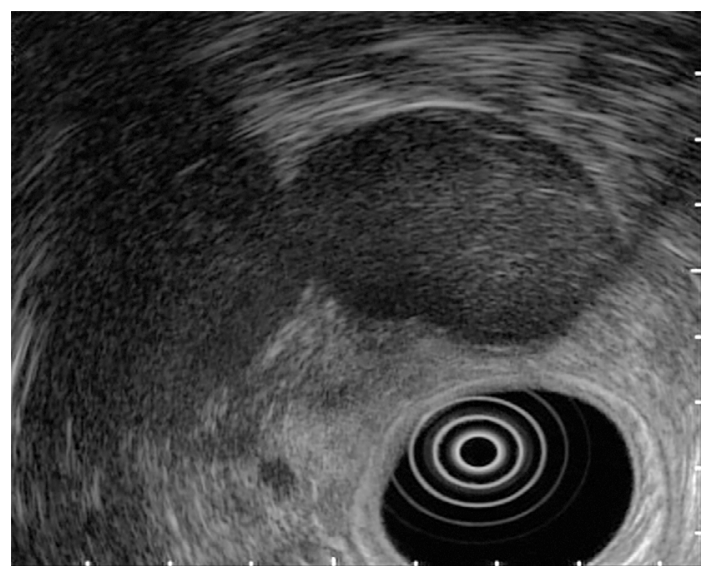

Figure 2. Endoscopic ultrasonography (EUS) showed a 40 mm unilocular cystic lesion within the pancreatic tail without an internal nodule.

of the pancreatic tail, which showed enhancement similar to the adjacent spleen (artery phase) (Fig. 1A), (portal phase) (Fig. 1B). An abdominal MRI showed T1 low / T2 high signal consistent with the pancreatic cystic lesion. Endoscopic ultrasonography (EUS) showed a $40 \mathrm{~mm}$ unilocular cystic lesion within the pancreatic tail without an internal nodule (Fig. 2). Endoscopic retrograde pancreaticocholangiography (ERCP) showed neither any papillary dilation nor mucus discharge. The cystic lesion did not communicate with the main pancreatic duct. The pancreatic juice cytology showed no malignant cells. Given that cystic neoplasms including MCN were included in the differential diagnosis, we proceeded with resection by a laparoscopic spleen-preserving distal pancreatectomy. The gross pathology showed a cystic lesion measuring $50 \times 40 \mathrm{~mm}$ in size with a hyalinized fibrous capsule. The cyst was filled with serous fluid without internal bleeding, necrosis or calcifications. In the wall of the cyst, there were brown spleen-like organizations (arrow) (Fig. 3A). On histopathology the cyst wall showed a thin multilayered squamous epithelium (arrow head), with small patches of splenic epithelium (arrow) [Hematoxylin and Eo$\sin (\mathrm{H} \& \mathrm{E})$ staining, $\times 4]$ (Fig. 3B), (HE staining, $\times 40)$
(Fig. 3C). This constellation of these findings was consistent with an epidermoid cyst of an intrapancreatic accessory spleen. Her postoperative course was uneventful and she was discharged on the 14th day after the operation. The tumor marker CA19-9 normalized after the operation.

\section{Case 2}

A woman in her 60s had discomfort in her back. She had been pointed out to have a cystic lesion in the tail of the pancreas by a medical examination from three years prior to this presentation. It was diagnosed to be an IPMN of the side branch which measured about $30 \mathrm{~mm}$ in diameter. On our examination by abdominal US the lesion had increased to a diameter of $35 \mathrm{~mm}$. Her medical history included appendectomy at 15 years of age. Laboratory data showed the serum CA19-9 level to be slightly elevated at $52.9 \mathrm{U} / \mathrm{mL}$. Abdominal US showed the multilocular cystic lesion to measure $35 \mathrm{~mm}$ in size and be located within the pancreatic tail. The borders were clearly demarcated and the lesion showed internal heterogeneity. Contrast enhanced abdominal CT revealed a multilocular cystic lesion, measuring $35 \mathrm{~mm}$ in size, within the pancreatic tail. The solid component in the dorsal portion was revealed and showed enhancement similar to the spleen (artery phase) (Fig. 4A), (portal phase) (Fig. 4B). An abdominal MRI showed a multilocular cystic lesion having a low T1 signal and a high T2 signal. EUS showed a $35 \mathrm{~mm}$ multilocular cystic lesion within the pancreatic tail with an internal nodule (Fig. 5). ERCP views showed no papillary orifice dilatation or mucus. The cystic lesion was not in continuity between the pancreatic duct and the cyst. A cytologic examination of pancreatic juice showed no malignant cells. Given that cystic neoplasms including IPMN were included in the differential diagnosis, we proceeded with resection by a laparoscopic distal pancreatectomy. The gross appearance showed a multi cystic lesion measuring $35 \mathrm{~mm}$ in size with a hyalinized fibrous capsule. The serosity was accumulated in the lumen (Fig. 6A). The histopathological views were similar to case 1 (Fig. 6B and C) and the final diagnosis was an epidermoid cyst of an intrapancreatic accessory spleen. The progress 

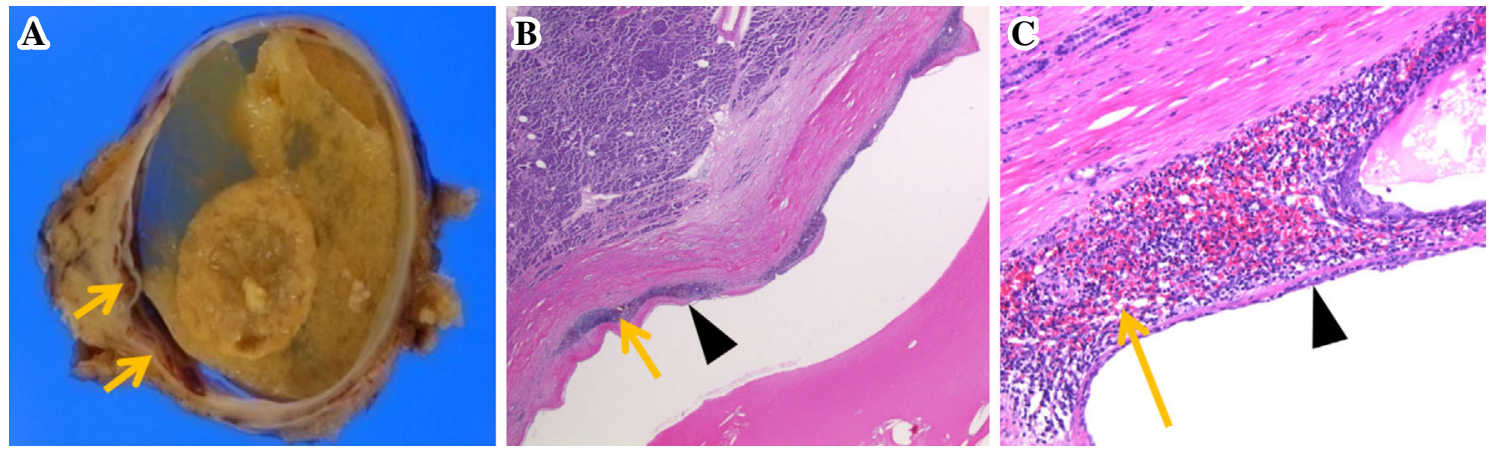

Figure 3. (A) Gross pathology showed a cystic lesion measuring $50 \times 40 \mathrm{~mm}$ in size with a hyalinized fibrous capsule. The cyst was filled with serous fluid without internal bleeding, necrosis or calcifications. In the wall of the cyst, there were brown spleen-like organizations (arrow). On histopathology the cyst wall showed a thin multilayered squamous epithelium (arrow head), with small patches of splenic epithelium (arrow) [Hematoxylin and Eosin (H\&E) staining, ×4] (B), (H\&E staining, ×40) (C).
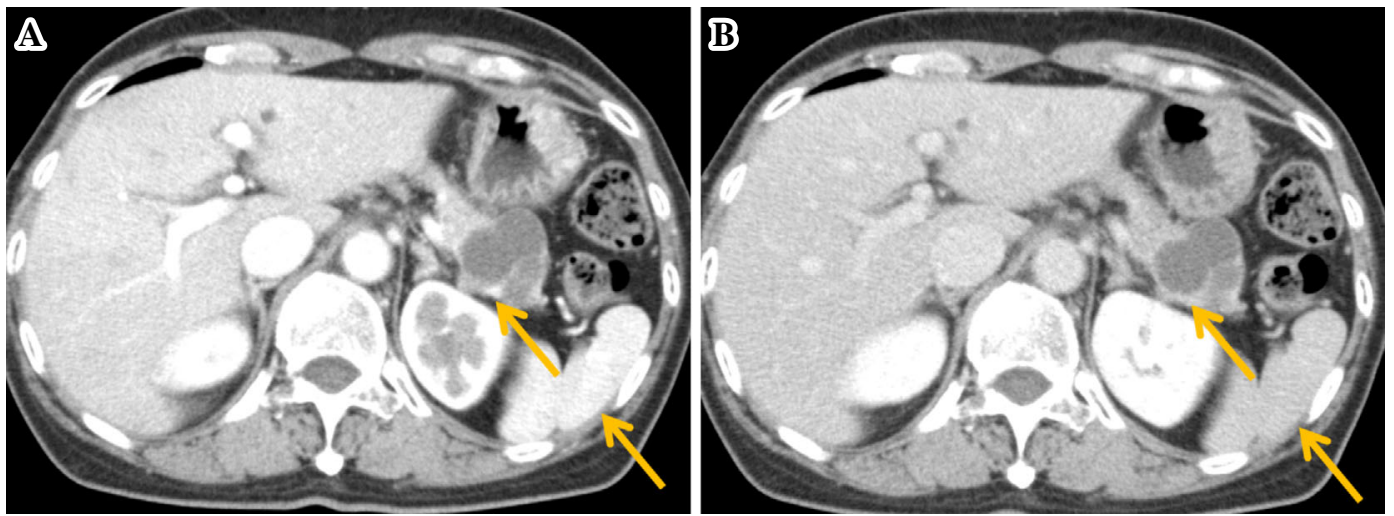

Figure 4. Contrast enhanced abdominal CT revealed a multilocular cystic lesion, measuring $35 \mathrm{~mm}$ in size, within the pancreatic tail. The solid component in the dorsal portion was revealed and showed enhancement similar to the spleen (arrows) (artery phase) (A), (portal phase) (B).

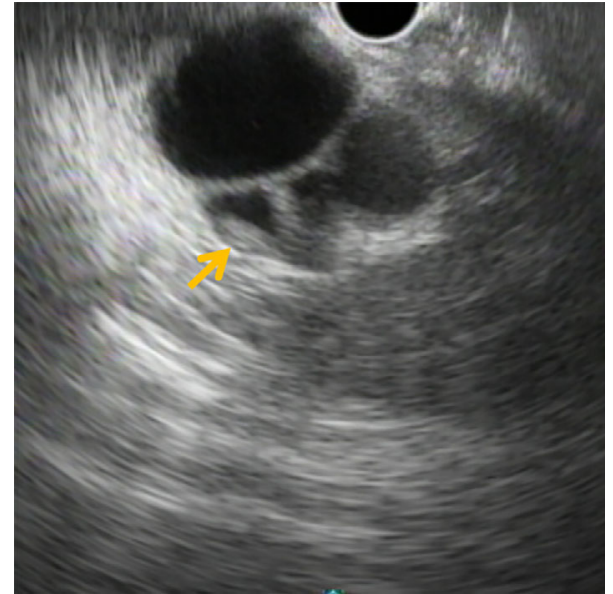

Figure 5. EUS showed a $35 \mathrm{~mm}$ multilocular cystic lesion within the pancreatic tail with an internal nodule.

was good, and she left the hospital on the 14th day after the operation. The tumor markers normalized after the operation.

Discussion

In the pancreas, 3 types of morphologically similar lesions may present as "squamous cysts": lymphoepithelial cysts, dermoid cysts, and epidermoid cysts within intrapancreatic accessory spleens. In our cases, a thin stratified squamous epithelium layer existed inside the wall, and a spleen-like organization was recognized under epithelial tissue (3). This was consistent with an epidermoid cyst of an intrapancreatic accessory spleen of the pancreas. Halpert et al. reported 346 accessory spleens of which 291 cases $(10.8 \%)$ were recognized in 2,700 autopsies, including 215 $(62.1 \%)$ accessory spleens occurred around the splenic hilum and $78(22.5 \%)$ occurred within the tail of the pancreas (2). Because the spleen develops from the mesoderm, and has no lumen, a concomitant cyst within this tissue is rare $(0.5-2.0 \%)$. Therefore, an epidermoid cyst of an intrapancreatic accessory spleen is very rare. There are various hypotheses regarding the mechanisms through which an epidermoid cyst can arise within an accessory spleen. One possibility is that the cyst wall arises from an aberrant ectopic 

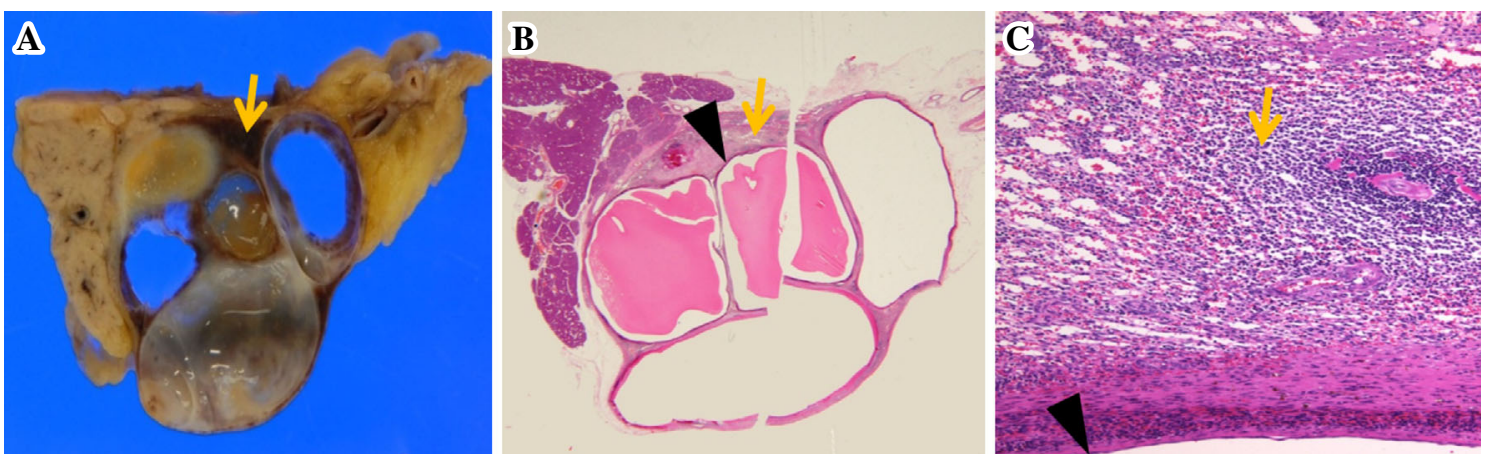

Figure 6. (A) The gross appearance showed a multi cystic lesion measuring $35 \mathrm{~mm}$ in size having the hyalinized fibrous capsule. The serosity was accumulated in the lumen. In the wall of the cyst, there were brown spleen-like organizations (arrow). On histopathology, the cyst wall showed a thin multilayered squamous epithelium (arrow head), with small patches of splenic epithelium (arrow) [Hematoxylin and Eosin (H\&E) staining, x1] (B), (H\&E staining, ×40) (C).

endoblast organization within the developing mesodermal tissue $(4,5)$. Another possibility is that the cyst wall is not mesothelial, but rather of pancreatic ductal origin because they are often negative for the mesothelial markers calrectinin, vimentin, and CA125, but positive for ductal markers such as EMA and CA19-9 (6-8).

Reported epidermoid cysts within an intrapancreatic accessory spleen are extremely rare, with the first described case in 1980 by Davidson et al. (9). In 2013, Hong et al. summarized their experience with 31 cases of epidermoid cyst within an intrapancreatic accessory spleen (10). These cases involved 15 males and 16 females, with ages ranging from 12-70 years (mean, 46 years). All cysts were located in the pancreatic tail. While 16 patients were asymptomatic, the other 14 patients demonstrated multiple discordant symptoms including weight loss, nausea, vomiting, abdominal pain and discomfort, back pain, epigastric pain and fever. A histological analysis from this series revealed that the cysts could be either solitary or multilocular, lined with a keratinized or non-keratinized stratified squamous epithelium or a cuboidal epithelium, and in some cases exhibiting a mixed-form epithelium. An elevation of the serum CA 19-9 level was observed in 10 cases, hence the difficulty in preoperatively differentiating between an epidermoid cyst within an intrapancreatic accessory spleen and pancreatic malignancy during the clinical workup. Distal pancreatectomy was performed for a preoperative diagnosis of pancreatic cystic neoplasms including possible malignant tumors. Only five cases were managed laparoscopically (10-14). There has been significant progress in the laparoscopic techniques for pancreatic masses and it is thought to be an effective method of surgical management in cases of pancreatic and splenic cystic lesions, avoiding the morbidity of open surgery (15). For unspecified tumors, such as our described cases, which appear to be benign, but malignancy cannot be completely ruled out, a laparoscopic approach is the best way to avoid the drawbacks of open surgery including increased pain, prolonged hospitalization, and a poor cosmetic result. Our patients were both concerned with a good cosmetic result (16). It has been reported that the serum CA 19-9 level markedly decreased to within the normal range following surgery in patients undergoing resection for an epidermoid cyst within an intrapancreatic accessory spleen $(17,18)$. It is also noteworthy that the majority of patients with this disease are of Asian origin (28/36 patients, $77.7 \%$ ), suggesting a possible ethnic or genetic tendency (19-21).

It is important to distinguish between the cystic and solid parts of the lesion, so that a correct diagnosis can be made preoperatively $(22,23)$. The solid part shows the same echogenicity as the spleen on abdominal US and EUS. In addition, contrast CT and MRI show that the solid component is similar to the spleen. Further diagnostic workup could include the use of superparamagnetic iron oxide (SPIO) contrast MRI to diagnose intrapancreatic accessory spleen (24). When the SPIO contrast media are given by phleboclysis, $80 \%$ is taken into the reticuloendothelial cells (Kupffer cell) within the liver, and the remainder is taken up by the spleen. Because SPIO has a very strong T2 shortening effect, a diagnosis of intrapancreatic accessory spleen can be improved by observing this effect in relation to the liver and spleen.

Incorporating the available diagnostic modalities to identify this lesion preoperatively could prevent unnecessary operations. If we notice the finding that splenic features exist in the wall of pancreatic tail cyst before operation, we can diagnose this disease and continue with the follow-up observations. To prevent any unnecessary surgical intervention, it is important that we recognize the epidermoid cyst of an intrapancreatic accessory spleen as the differential diagnosis of pancreatic cyst. Because the solid parts of the cyst are usually thin, comparing their features with the spleen is difficult, thus contributing to the difficulty in distinguishing this entity from other cystic neoplasms of the pancreas. Most reported cases resulted in open distal pancreatectomy and splenectomy which in retrospect was too aggressive for this benign lesion (11). We chose an operation through informed consent that would encompass an adequate resection in the event the lesion was an MCN or IPMN. 
In conclusion, we herein reported two cases of an epidermoid cyst within an intrapancreatic accessory spleen after laparoscopic distal pancreatectomy. This procedure affords the advantages of a minimally invasive approach for the treatment of benign or low-grade malignant tumors located in the pancreatic body or tail. Although these lesions are rare, it is important to consider this disease in the differential diagnosis of pancreatic tail cystic lesions.

The authors state that they have no Conflict of Interest (COI).

\section{References}

1. Halpert B, Gyorkey F. Lesions observed in accessory spleens of 311 patients. Am J Clin Pathol 32: 165-168, 1959.

2. Halpert B, Alden ZA. Accessory spleens in or at the tail of the pancreas. A survey of 2700 additional necropsies. Arch Pathol 77: 652-654, 1964.

3. Adsay NV, Hasteh F, Cheng JD, Klimstra DS. Squamous-lined cysts of the pancreas: lymphoepithelial cysts, dermoid cysts (teratomas), and accessory-splenic epidermoid cysts. Semin Diagn Pathol 17: 56-65, 2000.

4. Ough YD, Nash HR, Wood DA. Mesothelial cysts of the spleen with squamous metaplasia. Am J Clin Pathol 76: 666-669, 1981.

5. Bürrig KF. Epithelial (true) splenic cysts. Pathogenesis of the mesothelial and so-called epidermoid cyst of the spleen. Am J Surg Pathol 12: 275-281, 1988.

6. Horibe Y, Murakami M, Yamao K, Imaeda Y, Tashiro K, Kasahara M. Epithelial inclusion cyst (epidermoid cyst) formation with epithelioid cell granuloma in an intrapancreatic accessory spleen. Pathol Int 51: 50-54, 2001.

7. Morohoshi T, Hamamoto T, Kunimura T, et al. Epidermoid cyst derived from an accessory spleen in the pancreas. A case report with literature survey. Acta Pathol Jpn 41: 916-921, 1991.

8. Sasou S, Nakamura S, Inomata M. Epithelial splenic cysts in an intrapancreatic accessory spleen and spleen. Pathol Int 49: 10781083, 1999.

9. Davidson ED, Campbell WG, Hersh T. Epidermoid splenic cyst occurring in an intrapancreatic accessory spleen. Dig Dis Sci 25: 964-967, 1980.

10. Hong R, Choi N, Sun K, Lim S, Han Y. Epidermoid cyst arising from an intrapancreatic accessory spleen: a case report and review of the literature. Oncol Letters 5: 469-472, 2013.

11. Itano O, Chiba N, Wada T, et al. Laparoscopic resection of an epidermoid cyst originating from intrapancreatic accessory spleen: report of a case. Surg Today 40: 72-75, 2010.
12. Iwasaki $\mathrm{Y}$, Tagaya N, Nakagawa A, et al. Laparoscopic resection of epidermoid cyst arising from an intrapancreatic accessory spleen: a case report with a review of the literature. Surg Laparosc Endosc Percutan Tech 21: 275-279, 2011.

13. Urakami A, Yoshida K, Hirabayashi Y, et al. Laparoscopy-assisted spleen-preserving pancreatic resection for epidermoid cyst in an intrapancreatic accessory spleen. Asian J Endosc Surg 4: 185-188, 2011.

14. Harris AC, Chaudry MA, Menzies D, Conn PC. Laparoscopic resection of an epidermoid cyst within an intrapancreatic accessory spleen: a case report and review article. Surg Laparosc Endosc Percutan Tech 22: 246-249, 2012.

15. Iacobone M, Citton M, Nitti D. Laparoscopic distal pancreatectomy: up-to-date and literature review. World J Gastroenterol 18: 5329-5337, 2012.

16. Wakasugi M, Tori M, Akamatsu H, et al. Laparoscopic distal pancreatectomy for multiple epithelial cysts in an intrapancreatic accessory spleen. A case report and review of literature. JOP 14: 636-641, 2013.

17. Watanabe H, Yamaguchi Y, Ohtsubo K, et al. Epidermoid cyst of the intrapancreatic accessory spleen producing CA19-9. Digestive Endoscopy 16: 222-248, 2004.

18. Higaki K, Jimi A, Watanabe J, Kusaba A, Kojiro M. Epidermoid cyst of the spleen with CA19-9 or carcinoembryonic antigen productions: report of three cases. Am J Surg Pathol 22: 704-708, 1998.

19. Zhang Z, Wang JW. An epithelial splenic cyst in an intrapancreatic accessory spleen. A case report. JOP 10: 664-666, 2009.

20. Reiss G, Sickel JZ, See-Tho K, Ramrakhiani S. Intrapancreatic splenic cyst mimicking cystic neoplasm diagnosed by EUS-FNA. Gastrointest Endosc 70: 557-558, 2009.

21. Zavras N, Machairas N, Foukas P, Lazaris A, Patapis P, Machairas A. Epidermoid cyst of an intrapancreatic accessory spleen: a case report and literature review. World J Surg Oncol 12: 92, 2014.

22. Itano O, Shiraga N, Kouta E, et al. Epidermoid cyst originating from an intrapancreatic accessory spleen. J Hepatobil Pancreas Surg 15: 436-439, 2008.

23. Hu S, Zhu L, Song Q, Chen K. Epidermoid cyst in intrapancreatic accessory spleen: computed tomography findings and clinical manifestation. Abdom Imaging 37: 828-833, 2012.

24. Kim SH, Lee JM, Han JK, et al. MDCT and superparamagnetic iron oxide (SPIO)-enhanced MR findings of intrapancreatic accessory spleen in seven patients. Eur Radiol 16: 1887-1897, 2006.

The Internal Medicine is an Open Access article distributed under the Creative Commons Attribution-NonCommercial-NoDerivatives 4.0 International License. To view the details of this license, please visit (https://creativecommons.org/licenses/ by-nc-nd/4.0/).

(C) 2016 The Japanese Society of Internal Medicine http://www.naika.or.jp/imonline/index.html 